\title{
Does Gamified Educational Systems Change Students' Learning Behaviors? A Case Study with Postgraduate Students
}

\author{
Wilk Oliveira dos Santos ${ }^{1}$, Armando Toda ${ }^{1}$, Seiji Isotani ${ }^{1}$, Ig Ibert Bittencourt ${ }^{2}$ \\ ${ }^{1}$ Laboratory of Applied Computing in Education and Advanced Social Technology (CAEd) \\ Institute of Mathematical and Computer Sciences (ICMC) \\ University of São Paulo (USP) - São Carlos - SP - Brazil \\ ${ }^{2}$ Center of Excellence for Social Technologies (NEES) \\ Computer Institute (IC) \\ Federal University of Alagoas (UFAL) - Maceió - AL - Brazil \\ \{wilk.oliveira, armando.toda\}@usp.br, sisotani@icmc.usp.br, ig,iber@ic.ufal.br
}

\begin{abstract}
In the last few years, gamification studies have increased in the field of education and digital technologies, showing different results on the use of gamified educational systems in relation to students' learning aspects. These studies, however, often keep the focus on quantitative or qualitative approaches directly related to student learning, without considering students' perceptions of the gamification design and how the gamification design aspects of the system can influence positively the students' behavior change. Therefore, this study compared a gamified with a non-gamified version of an educational system, through a mixed (quantitative and qualitative) approach to identify if gamified educational systems are better than non-gamified educational systems to provide positive students' learning behavior change. The results confirmed that the gamified educational system was more effective than the non-gamified system.
\end{abstract}

\section{Introduction}

To target problems such as evasion, disengagement and demotivation in educational systems, recent studies have used game and gamification elements along with their activities [Battistella and von Wangenheim 2016, Challco et al. 2015, Monterrat et al. 2014]. They aim to decrease students' frustration and demotivation as well as to improve students' concentration, engagement, and learning [Cózar-Gutiérrez and Sáez-López 2016, Paiva et al. 2015b, Paiva et al. 2016]. These studies are implementing and evaluating the use of gamification in educational systems, raising the concept of gamified educational systems.

Recent results have shown that these systems can offer different ways for the students to perform the educational activities associated with gamification elements Hamari et al. 2014, Nah et al. 2014, Sousa Borges et al. 2014, Dicheva and Dichev 2015]. In addition, gamified educational systems may provide a number of benefits to students, such as: increase their motivation [Cózar-Gutiérrez and Sáez-López 2016, Hanus and Fox 2015] and learning performance [Nah et al. 2014, Pedro et al. 2015]. These effects aroused the interest and investment in gamification by many institutions. 
However, other studies are showing that, at several cases, the use of gamification in an educational context (especially gamified educational systems) does not necessarily improve the students' motivation, engagement, and learning [Hamari et al. 2014, Orji 2014, Orji et al. 2014, Toda et al. 2018]. These results are arousing the attention of the community for the need of deeper researches to identify when and how the use of gamification is effective to improve the students' satisfaction and to propose solutions to provide, indeed, a better gamification design which might impact on learning performance [Hamari et al. 2016, Hamari et al. 2014].

With the aim of identifying if gamified educational systems have a more capacity than non-gamified educational systems to positively change students' behavior in terms of learning habits, we conducted a qualitative and quantitative study based on known methods of Human-Computer Interaction (HCI). Both methods pointed that the gamified educational system influenced students more positively to change their learning habits than the non-gamified educational system. It was also possible to notice that the students preferred some gamification elements more than others.

\section{Background}

This section aims to present our study background, that is evaluation of Human-Computer Interaction in gamified educational systems. We also aim to present our main related works.

\subsection{Evaluation of Human-Computer Interaction in gamified educational systems}

Throughout history, many have championed the use of play, games, and game-inspired design to improve the human condition [Nacke and Deterding 2017]. Recently, gamification has become a popular method for enriching information technologies, presenting the use of gamification in different types of systems, including educational systems [Hamari et al. 2014].

The term "gamification", according to [Hamari et al. 2016] was first used in 2008 , in a blog posted by Brett Terrill, describing the word as "taking game mechanics and applying them to other web properties to increase engagement". However, the concept of gamification has been commonly adopted by the digital media industry [Deterding et al. 2011]. The first documented use dates to 2008 by Paharia [Paharia 2010], but the term has not been widespread adopted before the second half of 2010 [Deterding et al. 2011].

In the recent years, different studies were conducted with the objective of evaluating the effects of gamification from an HCI perspective [Hamari et al. 2014, Nah et al. 2014, Dicheva et al. 2015]. In general, these studies often uses quantitative approaches based on questionnaires of scales for usability test OOrji 2014, Klock et al. 2017], or qualitative approaches based on open questions [Orji et al. 2018, Orji et al. 2014], leaving aside other methods of the HCI that can aid in a more direct evaluation of the perception of the users concerning the design of the system.

\subsection{Related Works}

Persuasive systems tend to influence and encourage the user to adopt new behaviours and change undesirable behaviour [Fogg 2002]. Based on that, in the last years, some studies 
has been conducted in order to evaluate how gamified persuasive systems can change undesirable behaviour. Recently, [Klock et al. 2017] evaluated the usability and the user experience of gamified educational systems, using a heuristic evaluation as guideline in 10 different gamified educational systems. From they study, four systems with more problems were selected to perform usability testing in the laboratory and to fill out a questionnaire by participants.

Besides, [Orji et al. 2018] conducted a large-scale study of 543 participants to investigate how different gamification user types responded to ten persuasive strategies depicted in storyboards representing persuasive gameful health systems to change the habits of alcoholic people in relation to their consumption of beverages. The results found by [Orji et al. 2018] reveal that people's gamification user types play significant roles in the perceived persuasiveness of different strategies.

The previously mentioned studies generally kept their focus on the evaluation of usability of the system, as well as on the evaluation of change of behavior by the users, however, is also important identifying if gamified educational systems can also help to positively change students' learning habits, also, in both studies, methods such as scales and evaluation of specialties were used, leaving in open the importance of conducting studies using different methods of HCI to identify the perception of students regarding the gamification elements.

\section{Study Design and Methods}

Our study had as main objective to investigate if gamified educational systems are better than the non-gamified educational systems to change students' learning behaviour. To achieve our objective, we first chose a gamified educational system to use in our study. After a comparison between nine different gamified educational systems we choose the system MeuTutor due to its group of gamification elements being one of the most used in the literature [Nah et al. 2014, Dicheva et al. 2015] and its design of gamification which had been validated in previous studies [Paiva et al. 2015a, Paiva et al. 2016, Santana et al. 2016]. For the non-gamified version of the system, we removed all of the gamification elements from the system with help of two gamification designers with expertise in the design of gamified educational systems.

Although it is a system that has already been validated and used in a real scenario, after modifying the system by removing the gamification elements (for the non-gamified version), we chose to conduct a new interface evaluation based on Nielsen's 10 heuristics [Nielsen and Molich 1990] interfaces web. This assessment was conducted by a specialist in usability and interface evaluation. After conducting the heuristic evaluation by the specialist, the activities that each study participant should follow in each version of the system were defined. The sequence of activities were defied in partnership with an HCI research specialist.

In the gamified system, the participant should start by choosing an avatar, before accessing the initial screen, which contains their chosen avatar, a progress bar, and their points. In this screen, the user should choose the Mathematics discipline. In the next screen, the user visualizes an activity tree, which presents the progress bar. In this screen, the user should choose the first available activity. In the next screen, the user should respond to an activity, after the response, the user would receive feedback on his response. 
Step 1

Choose the avatar

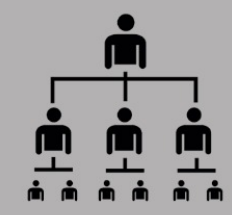

Step 4

Solve the Activity

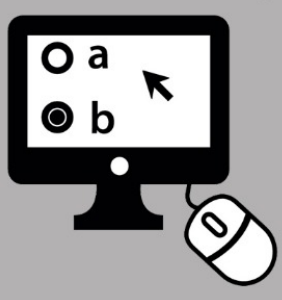

Step 2

Choose Domain

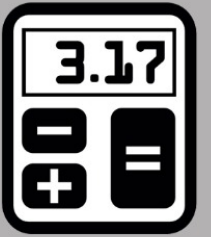

Step 5

Receive Feedback

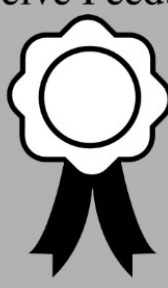

Step 3

Choose the Activity

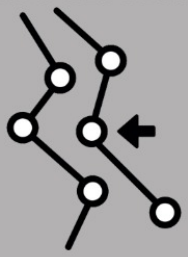

Step 6

Go to profile statistics

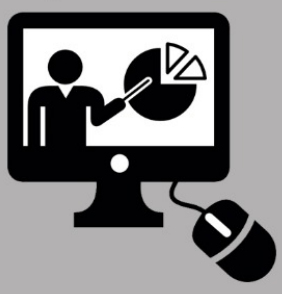

Figure 1. Gamified system screen sequence

Finally, the users should access the profile screen, where they would be shown the elements of gamification, ranking, trophies, avatar, points, levels and progress bar. The Figure 1 presents the screens of the gamified system organized according to the order of the proposed activities.

In the non-gamified version of the system, the user already starts on the system's home screen because the avatar element has been removed. Then the user should choose the Mathematics discipline. In the next screen, a tree is presented to the participant, who again must choose the first activity available. In the next screen, the user should respond to the activity, where, after their answer, they would receive a feedback according to their response. Finally, the user should access the profile screen. In all screens, only the general contents of the system (without the gamification elements) would be presented to the participants.

\subsection{Method}

In order to conduct this study we choose a mixed method, approach (quantitative and qualitative). For the quantitative approach we used a validated scale for assessing perceived persuasiveness. The scale was adapted from [Drozd et al. 2012] and has been used in other persuasive technologies research [Orji et al. 2014, Busch et al. 2016, Orji et al. 2018]. The scale aims at evaluate four constructs: influence, persuasion, relevance and learning habits. These constructs are represented in the questions as follows: i) The system would influence me to study.; ii) The system would be convincing to study.; iii) The system would be personally relevant for my study.; $i v$ ) The system would make me reconsider my learning habits. The questions were measured using participant agreement with a 7- point Likert scale [Likert 1932] ranging from " 1 = Strongly disagree" to "7 = Strongly agree". We also included open-ended questions that allowed participants to provide qualitative comments to justify their ratings of each strategy.

For the qualitative approach we used the Thinking Aloud Protocol, which is a 
protocol used to gather data in usability testing in product design and development, in psychology and a range of social sciences [Lewis 1982], it is also commonly used in HCI studies. The protocol aims to make participants "thinking aloud" as they are performing a set of specified tasks. Participants are asked to speak freely whatever it is in their mind as they complete the task. This might include what they are looking at, thinking, doing, and feeling, giving observers insight into the participant's cognitive processes. In our case, the participants accepted to have their speeches and actions recorded in video and audio for future analysis of the researchers.

\subsection{Participants, Data collection and Analysis}

Our study has nine participants (seven males and two females) postgraduate students in the field of Computer Sciences, all of them have experience in the use of educational systems (seven also had previous contact with gamified educational systems). Data were collected individually from each participant. Initially, a random algorithm was executed to know which version of the system (gamified or non-gamified) the participant would use first. Participants then read and accepted the term of participation, and used the system following the Thinking Aloud Protocol. Finally, each participant answered the survey questionnaire and repeated the same process with the other version of the system.

\section{Results}

Regarding the results of our quantitative study, it is possible to notice that for all cases, the gamified educational system presented better results in comparison with the nongamified system. These results indicate that gamified educational systems can help students change their study-related habits, for instance, by helping a student spend more time using an educational learning system or interacting with peers in an environment of this type for the better. At the same time, these results also corroborate with the existing literature on gamification, which indicates at first glance, gamification always tends to have positive effects on most students in educational settings [Hamari et al. 2014, Hamari 2015, Paiva et al. 2016]. The Figure 2 summarizes these results.

To compare if the gamified system presents significantly different results in relation to the non-gamified educational system in each of the observed issues, for example, if the gamified system is significantly better than the non-gamified system for influence the students to study, we used the statistical method $t$-test [Kim 2015]. The Table 1]present the mean of each issue measured in the questionnaire and the $t$-test results comparing the mean of each issue. The application of the test, considering $\mathrm{a} \geq 0.05$ level of significance and $95 \%$ confidence level

The $t$-test result indicates that in all evaluated items, the gamified version presented higher results than the non-gamified version, however, considering the $\geq 0.05$ level of significance, for three of the four evaluated issues, the difference was not statistically significant. Only in the item that measured if the system would convince the student to study, the difference was statistically significant ( $p$-value of 0.038 ). This result points to the fact that the gamified system was always better, however, not always, the difference was statistically significant.

Regarding the results of our qualitative study, it was also possible to perceive that most of the students felt more excited about the gamified version of the system. The main 

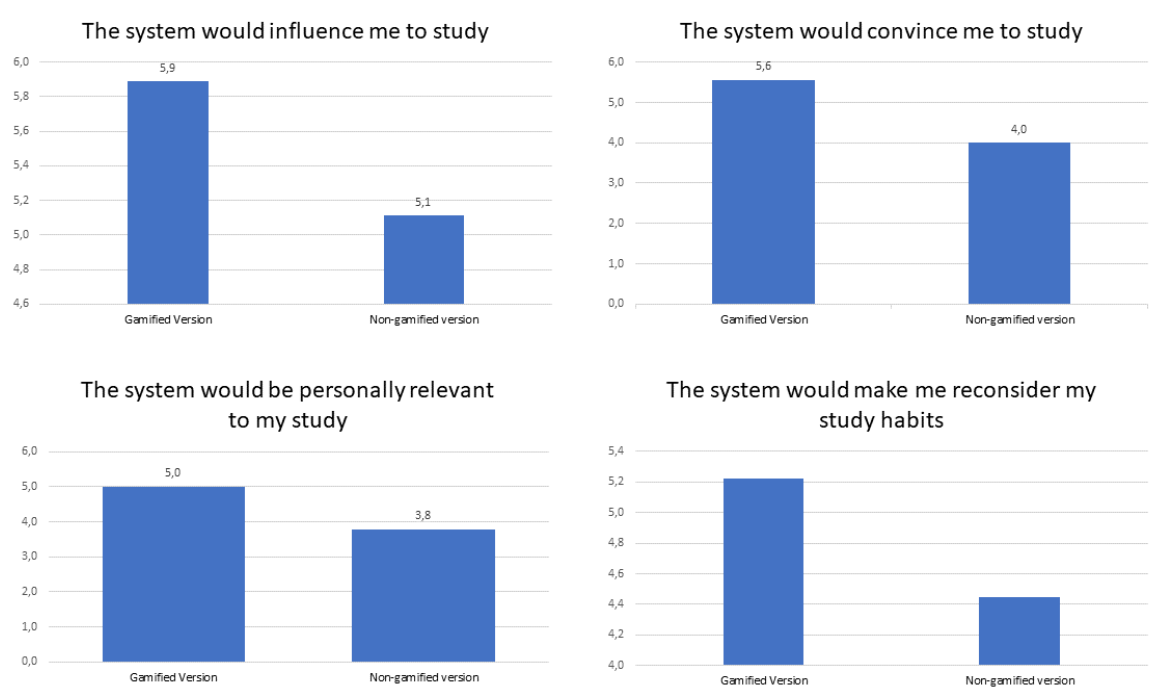

Figure 2. Results

Table 1. T-test Results

\begin{tabular}{llll}
\hline \multicolumn{1}{c}{ Questions } & \multicolumn{2}{c}{ Mean } & \multirow{2}{*}{ t-test } \\
\cline { 2 - 3 } & Gamified system & Non-gamified system & 0.219 \\
\hline $\begin{array}{l}\text { The system would } \\
\text { influence me to study }\end{array}$ & 5.889 & 5.111 & $\mathbf{0 . 0 3 8}$ \\
\hline $\begin{array}{l}\text { The system would } \\
\text { convince me to study }\end{array}$ & 5.555 & 4.000 & 0.127 \\
\hline $\begin{array}{l}\text { The system would } \\
\text { be personally relevant } \\
\text { to my study }\end{array}$ & 5.000 & 3.777 & 0.325 \\
\hline $\begin{array}{l}\text { The system would } \\
\text { make me reconsider } \\
\text { my study habits }\end{array}$ & 5.222 & 4.444 & \\
\hline
\end{tabular}

element of design that attracted the attention of the participants was the "avatars". Three participants drew attention to avatars, spending most of the time on the system in choosing these avatars. These participants found some specific avatar and said that avatar was more like yourself, or was more beautiful, and so would choose that avatar. These participants also realized that the avatar evolved while the system was being used.

During the evaluation of the non-gamified educational system, a few more observations made by participants drew the attention of the specialist that applied the Think Aloud Protocol. The first of these was the fact that the students found a "non-existent gamification element" and said that the best part of the non-gamified system was the content tree and the feedback given after the activity response. When asked why they chose those design elements, the students said it was because they looked like game design elements.

Our results corroborate with the existing literature as it confirms the results of 
recent studies that show that gamified educational systems may be more effective than non-gamified educational environments in terms of learning aspects (e.g., engagement [Barata et al. 2013] and motivation [Hanus and Fox 2015]). At the same time, our results show that some gamification elements may draw more attention than others in students (in our case, the avatars were better perceived), as well as other design elements, which in many cases are not associated with gamification (i.e., content tree and feedback) can also draw the attention of students.

The different student's perceptions regarding the gamification elements confirm the perspectives of recent studies, for example [Orji et al. 2018] and [Lavoué et al. 2018], which show that students have different preferences regarding the gamification elements. The reason for these different perceptions may be related to the fact that people have different gamer types [Nacke et al. 2014], and according to their gamer type, can be motivated or unmotivated by different types of elements [Santos et al. 2018b]. This result also draws attention to the importance of personalizing gamification and conducting studies that investigate how personalized gamification influences students' learning and their behavior change in gamified educational systems.

\subsection{Limitations}

As the study has the active participation of humans, it was also prone to a number of limitations, such as: it is possible that they were bored or tired while answering the survey; the participants of the study are representative only in an academic context (post-graduate students); some constructs may not be measured by the questions and protocol adopted; and the number of participants is limited, needing to be increased in future studies to to obtain more generalized results. At the same time, the questionnaire used was based on a questionnaire originally published in English and translated into Portuguese. Thus, the questionnaire may not accurately reproduce the original questionnaire. In order to mitigate this limitation, a cross-translation method was used, where the translation of two professionals was compared and a third expert decided on the best translation for the questionnaire.

\section{Concluding Remarks, Discussion and Future Works}

This study compared two versions of the same educational system (gamified and nongamified) with the aim of identifying whether thriving educational systems have a greater capacity to change students' learning behavior. To do this, we used a quantitative approach based on a validated questionnaire commonly used in $\mathrm{CHI}$ research and a qualitative approach, based on a validated protocol commonly used in $\mathrm{CHI}$ researches.

The main results confirmed that the gamified educational system was more effective than the non-gamified in the assessed aspects. This corroborates the gamification literature that gamification is effective in short term outcomes, we also provide some empirical evidence on gamification as a behavioral change technology, which is also something debated but with little evidence in the literature [Seaborn and Fels 2014]. We also could provide some insight on the acceptance of the game elements by our sample, where the users were satisfied with the set of elements that were used, which is also something that the literature says it is important but lacking in recent studies [Dichev and Dicheva 2017].

Our study collaborates with current literature on gamification, corroborating with studies that show that gamification can help to positively change people's behavior. In 
future studies, we aim to conduct a similar large-scale study with elementary and middle school students. We also want to verify if this approach impacted on students' motivation, engagement, and flow experience [Santos et al. 2018a], by analyzing the system log and compare it to non-gamified system.

\section{References}

[Barata et al. 2013] Barata, G., Gama, S., Jorge, J., and Gonçalves, D. (2013). Engaging engineering students with gamification. In Games and Virtual Worlds for Serious Applications (VS-GAMES), 2013 5th International Conference on, pages 1-8. IEEE.

[Battistella and von Wangenheim 2016] Battistella, P. E. and von Wangenheim, C. G. (2016). Engaged: Engaged: A game development process for teaching computing. In Brazilian Symposium on Computers in Education, volume 27, page 380.

[Busch et al. 2016] Busch, M., Mattheiss, E., Reisinger, M., Orji, R., Fröhlich, P., and Tscheligi, M. (2016). More than sex: The role of femininity and masculinity in the design of personalized persuasive games. In International Conference on Persuasive Technology, pages 219-229. Springer.

[Challco et al. 2015] Challco, G. C., Mizoguchi, R., Bittencourt, I. I., and Isotani, S. (2015). Gamification of collaborative learning scenarios: Structuring persuasive strategies using game elements and ontologies. In International Workshop on Social Computing in Digital Education, pages 12-28. Springer.

[Cózar-Gutiérrez and Sáez-López 2016] Cózar-Gutiérrez, R. and Sáez-López, J. M. (2016). Game-based learning and gamification in initial teacher training in the social sciences: an experiment with minecraftedu. International Journal of Educational Technology in Higher Education, 13(1):2.

[Deterding et al. 2011] Deterding, S., Dixon, D., Khaled, R., and Nacke, L. (2011). From game design elements to gamefulness: defining gamification. In Proceedings of the 15th international academic MindTrek conference: Envisioning future media environments, pages 9-15. ACM.

[Dichev and Dicheva 2017] Dichev, C. and Dicheva, D. (2017). Gamifying education: what is known, what is believed and what remains uncertain: a critical review.

[Dicheva and Dichev 2015] Dicheva, D. and Dichev, C. (2015). Gamification in education: Where are we in 2015? In E-Learn: World Conference on E-Learning in Corporate, Government, Healthcare, and Higher Education, pages 1445-1454. Association for the Advancement of Computing in Education (AACE).

[Dicheva et al. 2015] Dicheva, D., Dichev, C., Agre, G., and Angelova, G. (2015). Gamification in education: a systematic mapping study. Journal of Educational Technology \& Society, 18(3):75.

[Drozd et al. 2012] Drozd, F., Lehto, T., and Oinas-Kukkonen, H. (2012). Exploring perceived persuasiveness of a behavior change support system: a structural model. In International Conference on Persuasive Technology, pages 157-168. Springer.

[Fogg 2002] Fogg, B. J. (2002). Persuasive technology: using computers to change what we think and do. Ubiquity, 2002(December):5. 
[Hamari 2015] Hamari, J. (2015). Do badges increase user activity? a field experiment on the effects of gamification. Computers in human behavior.

[Hamari et al. 2014] Hamari, J., Koivisto, J., and Sarsa, H. (2014). Does gamification work?-a literature review of empirical studies on gamification. In System Sciences (HICSS), 2014 47th Hawaii International Conference on, pages 3025-3034. IEEE.

[Hamari et al. 2016] Hamari, J., Shernoff, D. J., Rowe, E., Coller, B., Asbell-Clarke, J., and Edwards, T. (2016). Challenging games help students learn: An empirical study on engagement, flow and immersion in game-based learning. Computers in Human Behavior, 54:170-179.

[Hanus and Fox 2015] Hanus, M. D. and Fox, J. (2015). Assessing the effects of gamification in the classroom: A longitudinal study on intrinsic motivation, social comparison, satisfaction, effort, and academic performance. Computers \& Education, 80:152-161.

[Kim 2015] Kim, T. K. (2015). T test as a parametric statistic. Korean journal of anesthesiology, 68(6):540-546.

[Klock et al. 2017] Klock, A. C. T., de Borba, E. J., Gasparini, I., Lichtnow, D., Pimenta, M. S., and Rodriguez, G. (2017). Evaluation of usability and user experience regarding the gamification of educational systems. In Learning Technologies (LACLO), 2017 Twelfth Latin American Conference on, pages 1-8. IEEE.

[Lavoué et al. 2018] Lavoué, É., Monterrat, B., Desmarais, M., and George, S. (2018). Adaptive gamification for learning environments. IEEE Transactions on Learning Technologies.

[Lewis 1982] Lewis, C. (1982). Using the'thinking-aloud'method in cognitive interface design. Research Report RC9265, IBM TJ Watson Research Center.

[Likert 1932] Likert, R. (1932). A technique for the measurement of attitudes. Archives of psychology.

[Monterrat et al. 2014] Monterrat, B., Lavoué, É., and George, S. (2014). A framework to adapt gamification in learning environments. In European Conference on Technology Enhanced Learning, pages 578-579. Springer.

[Nacke et al. 2014] Nacke, L. E., Bateman, C., and Mandryk, R. L. (2014). Brainhex: A neurobiological gamer typology survey. Entertainment computing, 5(1):55-62.

[Nacke and Deterding 2017] Nacke, L. E. and Deterding, S. (2017). The maturing of gamification research.

[Nah et al. 2014] Nah, F. F.-H., Zeng, Q., Telaprolu, V. R., Ayyappa, A. P., and Eschenbrenner, B. (2014). Gamification of education: a review of literature. In International conference on hci in business, pages 401-409. Springer.

[Nielsen and Molich 1990] Nielsen, J. and Molich, R. (1990). Heuristic evaluation of user interfaces. In Proceedings of the SIGCHI conference on Human factors in computing systems, pages 249-256. ACM.

[Orji 2014] Orji, R. (2014). Design for behaviour change: a model-driven approach for tailoring persuasive technologies. $\mathrm{PhD}$ thesis. 
[Orji et al. 2014] Orji, R., Mandryk, R. L., and Vassileva, J. (2014). Selecting effective strategies for tailoring persuasive health games to gamer types.

[Orji et al. 2018] Orji, R., Tondello, G. F., and Nacke, L. E. (2018). Personalizing persuasive strategies in gameful systems to gamification user types. In Proceedings of the 2018 CHI Conference on Human Factors in Computing Systems, page 435. ACM.

[Paharia 2010] Paharia, R. (2010). Who coined the term "gamification". linea] Quora.; http://goo. gl/CvcMsi [Access: 15/11/17].

[Paiva et al. 2015a] Paiva, R., Barbosa, A., Batista, E., Pimentel, D., and Bittencourt, I. I. (2015a). Badges and xp: An observational study about learning. In Frontiers in Education Conference (FIE), 2015 IEEE, pages 1-8. IEEE.

[Paiva et al. 2016] Paiva, R., Bittencourt, I. I., Tenório, T., Jaques, P., and Isotani, S. (2016). What do students do on-line? modeling students' interactions to improve their learning experience. Computers in Human Behavior, 64:769-781.

[Paiva et al. 2015b] Paiva, R. O. A., Bittencourt, I. I., da Silva, A. P., Isotani, S., and Jaques, P. (2015b). Improving pedagogical recommendations by classifying students according to their interactional behavior in a gamified learning environment. In Proceedings of the 30th Annual ACM Symposium on Applied Computing, pages 233-238. ACM.

[Pedro et al. 2015] Pedro, L. Z., Lopes, A. M., Prates, B. G., Vassileva, J., and Isotani, S. (2015). Does gamification work for boys and girls?: An exploratory study with a virtual learning environment. In Proceedings of the 30th Annual ACM Symposium on Applied Computing, pages 214-219. ACM.

[Santana et al. 2016] Santana, S. J., Paiva, R., Bittencourt, I. I., Ospina, P. E., de Amorim Silva, R., and Isotani, S. (2016). Evaluating the impact of mars and venus effect on the use of an adaptive learning technology for portuguese and mathematics. In Advanced Learning Technologies (ICALT), 2016 IEEE 16th International Conference on, pages 31-35. IEEE.

[Santos et al. 2018a] Santos, W. O., Bittencourt, I. I., Isotani, S., Dermeval, D., Marques, L. B., and Silveira, I. F. (2018a). Flow theory to promote learning in educational systems: Is it really relevant? Brazilian Journal of Computers in Education, 26(02):29.

[Santos et al. 2018b] Santos, W. O., Bittencourt, I. I., and Vassileva, J. (2018b). Design of tailored gamified educational systems based on gamer types. In Anais dos Workshops do Congresso Brasileiro de Informática na Educação, volume 7, page 42.

[Seaborn and Fels 2014] Seaborn, K. and Fels, D. I. (2014). Gamification in Theory and Action: A Survey. Internatoinal Journal of Human-Computer Studies, 74:14-31.

[Sousa Borges et al. 2014] Sousa Borges, S., Durelli, V. H., Reis, H. M., and Isotani, S. (2014). A systematic mapping on gamification applied to education. In Proceedings of the 29th Annual ACM Symposium on Applied Computing, pages 216-222. ACM.

[Toda et al. 2018] Toda, A. M., Valle, P. H., and Isotani, S. (2018). The dark side of gamification: An overview of negative effects of gamification in education. In Communications in Computer and Information Science, volume 832, pages 143-156. Springer, Cham. 